Y. Morita

Nagoya Math. J.

Vol. 75 (1979), 177-193

\title{
ON THE RADIUS OF CONVERGENCE OF THE $p$-ADIG L-FUNCTION
}

\author{
YASUO MORITA
}

\section{Introduction}

In their paper [11], Kubota-Leopoldt constructed the $p$-adic $L$-function $L_{p}(s ; \chi)$ for each Dirichlet character $\chi$. This function $L_{p}(s ; \chi)$ is a meromorphic function on the open $\operatorname{dics}\left\{|s-1|<\left|q^{-1} p^{1 /(p-1)}\right|\right\}$ with a possible simple pole at $s=1$. In this paper, we would like to give an answer to the following question:

Question. Why is $L_{p}(s ; \chi)$ meromorphic only for $|s-1|<\left|q^{-1} p^{1 /(p-1)}\right|$ and how does $L_{p}(s ; \chi)$ behave outside this open disc?

It is well-known that the $p$-adic exponential function $\exp (z)=$ $\sum_{n=0}^{\infty} z^{n} / n$ ! converges exactly for $|z|<\left|p^{1 /(p-1)}\right|$ and satisfies the functional equation $\exp \left(p^{n} z\right)=\{\exp (z)\}^{p^{n}}$ for any integer $n \geqq 0$. Hence $\exp (z)$ can be extended to a multivalued analytic function on the whole plane. We shall see in this paper that the $p$-adic $L$-function $L_{p}(s ; \chi)$ behaves exactly like this.

We assume that $\chi$ is primitive, $\chi \neq \chi^{0}$. and $\chi(-1)=1$. Then we shall show that (1) the $p$-adic $L$-function $L_{p}(s ; \chi)$ can be extended to a multivalued analytic function on the whole plane (see $\S 1$ ), but $(2) L_{p}(s ; \chi)$ can not be extended to a univalent analytic function on a larger domain (see § 2).

The result of Kubota-Leopoldt was generalized by several mathematicians to the case of a totally real finite algebraic number field. Accordingly, we shall study the problem in this generalized case. The results in this case is:

Let $F$ be a totally real finite algebraic number field. Let $\chi$ be a

Received October 16, 1978.

This paper was written when the author was a member of the Sonderforschungsbereich "Theoretische Mathematik" in Bonn. 
character of the Galois group of a totally real finite abelian extension of $F$. Let $L_{p}(s ; \chi)$ be the $p$-adic $L$-function for the character $\chi$. Then $L_{p}(s ; \chi)$ can be extended to a multivalued analytic function on the whole plane $k$ (see $\S 1$ ) and the maximal domain of analyticity for $L_{p}(s ; \chi)$ is given in the following manner (see $\S 2$ ):

Let $P_{\infty}$ be the cyclotomic $Z_{p}$-extension of $Q$, and let $F_{\infty}=F \cdot P_{\infty}$. Let $q_{0}$ be the degree of $\mathrm{F} \cap P_{\infty}$ over $\boldsymbol{Q}$. For each integer $m$, let

$$
D_{m}=\left\{s \in k|| s|<| p^{-m} q_{0}^{-1} p^{1 /(p-1)} \mid\right\} .
$$

Theorem. The p-adic L-function $L_{p}(s ; \chi)$ can be extended to a onevalued analytic function on $D_{m}(m \geqq 0)$ iff $L(s ; \pi \chi)=L(s ; \chi)$ holds for any character $\pi$ of the Galois group Gal $\left(F_{\infty} / F\right)$ satisfying $\pi^{p^{m}}=\chi^{0}$. Furthermore, if $m$ is maximum with this property, then $L_{p}(s ; \chi)$ has no (onevalued) analytic continuation outside $D_{m}$ or $D_{m} \mid\{1\}$.

Further we shall study the condition of this theorem and give an example.

In Appendix, we shall show that the algebra of Krasner analytic functions is integrally closed in its quotient field.

\section{$\S 1$. Multivalued analytic continuation of $L_{p}(s ; \chi)$}

Let $p$ be a prime number, and let $\boldsymbol{Q}_{p}$ be the $p$-adic number field. Let $k$ be an algebraically closed field containing $\boldsymbol{Q}_{p}$ such that (1) the $p$-adic absolute value of $\boldsymbol{Q}_{p}$ can be extended to an absolute value | | of $k$ and (2) $k$ is complete with respect to $\mid$. Let $\overline{\boldsymbol{Q}}$ be the algebraic closure of the rational number field $\boldsymbol{Q}$, and we fix embeddings of $\overline{\boldsymbol{Q}}$ into the complex number field $C$ and our field $k$. For each positive integer $n$, let $\zeta_{n}$ be the primitive $n$-th root of unity in $k$ corresponding to $\exp (2 \pi \sqrt{-1}) \in C$.

Let $F$ be a totally real finite algebraic number field, $M$ a finite cyclic extension of $F$, $f$ the conductor of $M / F$, (a, $M / F)$ the Artin symbol. Let $\chi$ be a 1-dimensional faithful character of the Galois group Gal $(M / F)$. For any fractional ideal $\mathfrak{a}$ of $F$ prime to $\mathfrak{f}$, let

$$
\chi(\mathfrak{a})=\chi((\mathfrak{a}, M / F)) \text {. }
$$

For any element $\sigma$ of $\mathrm{Gal}(M / F)$, let

$$
\zeta_{M}(s ; \sigma)=\sum(N a)^{-s},
$$


where $\mathfrak{a}$ runs over all integral ideals of $F$ prime to $\mathfrak{f}$ satisfying $(\mathfrak{a}, M / F)=\sigma$. It is well-known that $(1) \zeta_{M}(s ; \sigma)$ can be analytically continued to the whole plane $C$, except a simple pole at $s=1$, and $(2) \zeta_{M}(-n, \sigma)$ is a rational number for any integer $n \geqq 0$. Let $S$ be the set of primes of $F$ above $p$. We define

$$
L(-n, \chi)=\sum_{\sigma \in G} \chi(\sigma) \zeta_{M}(-n, \sigma)
$$

and

$$
L_{S}(-n, \chi)=L(-n, \chi) \prod_{\mathfrak{p} \in S}\left(1-\chi(\mathfrak{p})(N \mathfrak{p})^{n}\right) .
$$

Let $q$ denote $p$ or 4 according as $p \neq 2$ or $p=2$. For each integer $n \geqq 0$, let $P_{n}$ be the unique subfield of $\boldsymbol{Q}\left(\zeta_{q p^{n}}\right)$ of degree $p^{n}$ over $\boldsymbol{Q}$, and put $P_{\infty}=\bigcup_{n=1}^{\infty} P_{n}$. Let $F_{n}=F \cdot P_{n}$ for $n=0,1, \cdots, \infty$. Then $\operatorname{Gal}\left(F_{\infty} / F\right)$ is topologically isomorphic to the additive group $Z_{p}$ of $p$-adic integers. Let $\gamma_{0}$ be a topological generator of this group.

Let $F^{\prime}=F\left(\zeta_{q}\right), F_{\infty}^{\prime}=F^{\prime} \cdot F_{\infty}$. Then $\operatorname{Gal}\left(F_{\infty}^{\prime} / F\right)$ is isomorphic to the direct product of $\operatorname{Gal}\left(F^{\prime} \mid F\right)$ and $\operatorname{Gal}\left(F_{\infty} / F\right)$. Let $\theta$ and $\kappa$ be the characters of $\mathrm{Gal}\left(F^{\prime} / F\right)$ and $\mathrm{Gal}\left(F_{\infty} / F\right)$ with values in $Z_{p}$ such that

$$
\sigma(\zeta)=\zeta^{\theta(\sigma) \kappa(\sigma)}
$$

for any $p$-power root $\zeta$ of unity and $\sigma \in \operatorname{Gal}\left(F_{\infty}^{\prime} / F\right)$. We extend them to characters of $\operatorname{Gal}\left(F^{\prime} \cdot M / F\right)$.

Let $\chi$ be as before, and let $\mathfrak{o}_{\chi}$ be the ring of integers of the field $\boldsymbol{Q}_{p}(\chi)$ obtained by adjoining the values of $\chi$ to $\boldsymbol{Q}_{p}$. We say that $\chi$ is exceptional if $\chi$ is a character of $\operatorname{Gal}\left(F_{\infty} / F\right)$. Then $\chi\left(\gamma_{0}\right)$ is a $p$-power root of unity.

Coates proved the following theorem under two assumptions $C(p)$ and $D(p)$ (cf. Coates-Sinnot [4], Coates [3]). Later these two assumptions: were proved by Deligne, Ribet and Cassou-Noguès (cf. Deligne-Ribet [7], Cassou-Nouguès [2]).

Theorem C. Let $F$ be a totally real finite algebraic number field, and let $\chi$ be a character of the above form. Then there exists a unique element $G(T ; \chi)$ of the quotient field of the formal power series ring $\mathfrak{o}_{x}[[T]]$ with the following properties:

(1) $G(T ; \chi)$ belongs to $\mathfrak{o}_{\chi}[[T]]$ if $\chi$ is not exceptional.

(2) $\left\{\chi^{-1}\left(\gamma_{0}\right)(1+T)-\kappa\left(\gamma_{0}\right)\right\} G(T ; \chi)$ belongs to $o_{\chi}[[T]]$ if $\chi$ is exceptional.

(3) $L_{p}(s ; \chi)=G\left(\kappa\left(\gamma_{0}\right)^{s}-1 ; \chi\right)$ satisfies 


$$
L_{p}(-n ; \chi)=L_{S}\left(-n ; \chi \theta^{1-n}\right)
$$

for any integer $n \geqq 0$.

It is easy to see from Coates' construction of $G(T ; \chi)$ that we have

Corollary. Let $\chi$ be an exceptional character, and let $\eta_{\pi}=\pi\left(\gamma_{0}\right)^{-1}$. Then

$$
G\left(\kappa\left(\gamma_{0}\right)^{s}-1 ; \chi \pi\right)=G\left(\eta_{\pi} \kappa\left(\gamma_{0}\right)^{s}-1 ; \chi\right) .
$$

We note that our $G(T ; \chi)$ is $G\left(T ; \chi^{-1} \theta\right)$ in Coates' notation.

Let $\theta$ and $\pi$ be as before. Then we note that (a) $\theta^{2}$ is trivial iff $F \supseteq Q\left(\zeta_{q}+\zeta_{q}^{-1}\right)$ and (b) $\kappa\left(\gamma_{0}\right) \equiv 1$ modulo $q p^{n}$ iff $F \supseteq P_{n}$. We also note that $L_{p}(s ; \chi) \neq 0$ iff $M$ is totally real, and hence iff $\chi((\xi))=1$ for any element $\xi$ of $F$ satisfying $\xi \equiv 1$ modulo $\mathfrak{f}_{0}$, where $\mathfrak{f}_{0}$ is the finite part of $\mathfrak{f}$.

Let $q_{0}=q p^{e}$ be the greatest power of $p$ satisfying $\kappa\left(\gamma_{0}\right) \equiv 1$ modulo $q_{0}$. Hence $\left|\kappa\left(\gamma_{0}\right)-1\right|=\left|q_{0}\right|$. For any non-negative integer $m$, let

$$
D_{m}=\left\{s \in k|| s|<| p^{-1} q_{0}^{-1} p^{1 /(p-1)} \mid\right\} \text {. }
$$

Let $\mathcal{O}\left(D_{m}\right)$, be the set consisting of all power series $f(z)=\sum_{n=0}^{\infty} a_{n} s^{n}\left(a_{n} \in k\right)$ which converge on $D_{m}$. It is known that $\mathcal{O}\left(D_{m}\right)$ is a domain of integrity and $\mathcal{O}\left(D_{m}\right)$ is integrally closed in its quotient field $\mathscr{M}\left(D_{m}\right)$.

Let

$$
\exp (z)=\sum_{n=0}^{\infty} \frac{z^{n}}{n !} \quad \text { and } \quad \log (z)=\sum_{n=1}^{\infty} \frac{(-1)^{n-1}}{n} z^{n}
$$

Then

LEMMA 1. $k\left(\gamma_{0}\right)^{p^{m}}=\exp \left\{p^{m} s \log \left(\kappa\left(\gamma_{0}\right)\right)\right\}$ is an element of $\mathcal{O}\left(D_{m}\right)$. Furthermore the equation

$$
T_{m}^{p^{m}}=\kappa\left(\gamma_{0}\right)^{p_{s}}
$$

of $T_{m}$ is irreducible over $\mathscr{M}\left(D_{m}\right)$. Hence any root $T_{m}$ of this equation generates over $\mathscr{M}\left(D_{m}\right)$ a cyclic extension of degree $p^{m}$.

Proof. Since $\exp (z)$ converges exactly for $|z|<\left|p^{1 /(p-1)}\right|$ and since

$$
\left|\log \left(\kappa\left(\gamma_{0}\right)\right)\right|=\left|q_{0}+\cdots\right|=\left|q_{0}\right|,
$$

the first assertion follows immediately. Furthermore it follows that $\kappa\left(\gamma_{0}\right)^{p \ell}=$ $\exp \left\{p^{\ell} s \log \left(\kappa\left(\gamma_{0}\right)\right)\right\}$ does not belong to $\mathcal{O}\left(D_{m}\right)$ for any integer $\ell<m$. Since 
$\mathcal{O}\left(D_{m}\right)$ is integrally closed in $\mathscr{M}\left(D_{m}\right)$ and since $\kappa\left(\gamma_{0}\right)^{p e}$ is integral over $\mathcal{O}\left(D_{m}\right)$, it follows that $\kappa\left(\gamma_{0}\right)^{p \ell}$ does not belong to $\mathscr{M}\left(D_{m}\right)$. Hence

$$
\kappa\left(\gamma_{0}\right)^{p^{m}} \notin\left\{\mathscr{M}\left(D_{m}\right)^{\times}\right\}^{p} .
$$

Therefore, by Kummer theory, the equation in the lemma is irreducible over $\mathscr{M}\left(D_{m}\right)$. Hence the lemma is proved.

Let $\mathcal{O}\left(D_{m}\right)$ be as in Lemma 1 , let $T_{m}$ be a root of the equation in Lemma 1. Let $\mathcal{O}\left(D_{m}\right)\left[T_{m}\right]$ be the ring generated over $\mathcal{O}\left(D_{m}\right)$ by $T_{m}$. It is a subring of the algebraic closure $\left.\overline{\mathscr{M}\left(D_{m}\right.}\right)$ of $\mathscr{M}\left(D_{m}\right)$. Then

Lemma 2. (1) $\mathcal{O}\left(D_{m}\right)$ can be naturally identified with the set consisting of all power series in $T_{m}^{p^{m}}-1$ which converge for $\left|T_{m}^{p^{m}}-1\right|<\left|p^{1 /(p-1)}\right|$. (2) $\mathcal{O}\left(D_{m}\right)\left[T_{m}\right]$ can be naturally identified with the set consisting of all power series in $T_{m}-1$ that converge for $\left|T_{m}-1\right|<\left|p^{1 /(p-1) p^{m}}\right|$.

Proof. Since

$$
T_{m}^{p^{m}}=\exp \left\{p^{m} s \log \left(\kappa\left(\gamma_{0}\right)\right)\right\}=1+p^{m} \log \left(\kappa\left(\gamma_{0}\right)\right) s+\cdots
$$

is an element of $1+p^{1 /(p-1)} Z_{p}\left[\left[p^{m} \log \left(k\left(\gamma_{0}\right)\right) p^{-1 /(p-1)} s\right]\right]$, there exists a formal power series $F(X)=X+\cdots \in Z_{p}[[X]]$ such that

$$
p^{m} \log \left(\kappa\left(\gamma_{0}\right)\right) p^{-1 /(p-1)} s=F\left(\left(T_{m}^{p^{m}}-1\right) p^{-1 /(p-1)}\right) .
$$

It follows that $s \in D_{m}$ iff $\left|T_{m}^{p^{m}}-1\right|<\left|p^{1 /(p-1)}\right|$. Hence the first assertion follows immediately.

Since $T_{m}^{p^{m}}-1=\prod_{\eta}\left(T_{m}-\eta\right)=\prod_{\eta}\left\{\left(T_{m}-1\right)+(1-\eta)\right\}$, where $\eta$ runs over all $p^{m}$ th roots of unity, and since $|1-\eta|<\left|p^{1 /(p-1) p^{m}}\right|$ for any such $\eta$, $\left|T_{m}^{p^{m}}-1\right|<\left|p^{1 /(p-1)}\right|$ holds iff $\left|T_{m}-1\right|<\left|p^{1 /(p-1) p^{m}}\right|$. Further $F\left(\left(T_{m}^{p^{m}}-1\right) p^{-1 /(p-1)}\right)$ can be expanded into a power series of $\left(T_{m}-1\right) p^{-1 /(p-1) p^{m}}$ with the coefficients in $Z_{p}$. Hence any element of $\mathcal{O}\left(D_{m}\right)$, and hence any element of $\mathcal{O}\left(D_{m}\right)\left[T_{m}\right]$, can be expressed as a power series in $T_{m}-1$ that converges for $\left|T_{m}-1\right|<\left|p^{1 /(p-1) p^{m}}\right|$.

Let $f\left(T_{m}\right)=\sum_{n=0}^{\infty} b_{n}\left(T_{m}-1\right)^{n}$ be a power series of $T_{m}-1$ that converges for $\left|T_{m}-1\right|<\left|p^{1 /(p-1) p^{m}}\right|$. Let $\eta$ be a primitive $p^{m}$ th root of unity. Then

$$
f\left(T_{m}\right)=\sum_{\alpha=0}^{p^{m}-1} f_{\alpha}\left(T_{m}\right) T_{m}^{\alpha}
$$

where 


$$
\begin{aligned}
f_{\alpha}\left(T_{m}\right) & =\frac{1}{p^{m}} \sum_{\beta=0}^{p^{m-1}} \eta^{\alpha-\beta} f\left(\eta^{\beta} T_{m}\right) T_{m}^{-\alpha} \\
& =\sum_{n=0}^{\infty} b_{n}\left\{\frac{1}{p^{m}} \sum_{\beta=0}^{p^{m}-1} \eta^{\alpha-\beta}\left(\eta^{\beta} T_{m}-1\right)^{n} T_{m}^{-\alpha}\right\} .
\end{aligned}
$$

Since the sum in \{\} of (1.13) is a polynomial in $T_{m}^{p^{m}}$, we can write it as $\sum c_{\ell}^{(n)}\left(T_{m}^{p^{m}}-1\right)^{\ell}$. Then, taking the supremum for $\left|T_{m}-1\right|=r\left(\left|p^{1 /(p-1) p^{m-1}}\right|<\right.$ $\left.r<\left|p^{1 /(p-1) p^{m}}\right|\right)$, we obtain

$$
\left|\frac{1}{p^{m}} r^{n}\right| \geqq \operatorname{Max}_{\ell}\left|c_{\ell}^{(n)} r^{p^{m} \ell}\right|
$$

(cf. e.g. Krasner [10], p. 138). Therefore $f_{\alpha}\left(T_{m}\right)$ can be expressed as a power series in $T_{m}^{p^{m}}-1$ which converges for $\left|T_{m}^{p^{m}}-1\right|<\left|p^{1 /(p-1)}\right|$. It follows from the first assertion that $f_{\alpha}\left(T_{m}\right)$ can be expressed as a power series in $s$ which converges for $s \in D_{m}$. Therefore $f\left(T_{m}\right) \in \mathcal{O}\left(D_{m}\right)\left[T_{m}\right]$, which completes the proof of Lemma 2.

CoRollary. Let $f(T)=\sum a_{n} T^{n}$ be a formal power series of $T$ with the coefficients in $k$ such that $\left|a_{n}\right| \leqq 1(n=0,1, \cdots)$. Then (1) $f\left(k\left(\gamma_{0}\right)^{s}-1\right)$ is an element of $\mathcal{O}\left(D_{0}\right)$ and $(2) f\left(\kappa\left(\gamma_{0}\right)^{s}-1\right)$ can be extended to an element of $\mathcal{O}\left(D_{m}\right)\left[T_{m}\right]$ for any integer $m \geqq 0$.

Proof. Since

$$
\kappa\left(\gamma_{0}\right)^{s}-1 \in p^{1 /(p-1)} Z_{p}\left[\left[\log \left(\kappa\left(\gamma_{0}\right)\right) p^{-1 /(p-1)} s\right]\right],
$$

$f\left(\kappa\left(\gamma_{0}\right)^{s}-1\right)$ is a formal power series in $\log \left(\kappa\left(\gamma_{0}\right)\right) p^{-1 /(p-1)} s$ with $p$-integral coefficients. Hence $f\left(\kappa\left(\gamma_{0}\right)^{s}-1\right)$ gives an element of $\mathcal{O}\left(D_{0}\right)$.

Since $f(T)$ is a formal power series of $T$ with $p$-integral coefficients, $f(T-1)$ converges for $|T-1|<1$. Hence, by the assertion (2) of Lemma $2, f\left(T_{m}-1\right)$ is an element of $\mathcal{O}\left(D_{m}\right)\left[\left[T_{m}\right]\right]$. Since $T_{m}$ is an extension of $\kappa\left(\gamma_{0}\right)^{s}$ to an element of the algebraic closure $\overline{\mathscr{M}\left(D_{m}\right)}$ of $\mathscr{M}\left(D_{m}\right), f\left(T_{m}-1\right)$ may be regarded as an extension of $f\left(\kappa\left(\gamma_{0}\right)^{s}-1\right)$ to an element of $\overline{\mathscr{M}\left(D_{m}\right)}$. Hence the corollary is proved.

Remark. Let $f\left(T_{m}-1\right)$ be as above. Then it is a root of an irreducible monic polynomial with coefficients in $\mathscr{M}\left(D_{m}\right)$. Furthermore $f\left(\kappa\left(\gamma_{0}\right)^{s}-1\right)$ satisfies this equation for $s \in D$. In this paper, we use the word an $e x$ tension in this sense (cf. Krasner [10]). It is easy to see that any extension of an element of $\mathscr{M}\left(D_{0}\right)$ to an element of $\left.\overline{\mathscr{M}\left(D_{m}\right.}\right)$ is unique up to automorphisms of $\overline{\mathscr{M}\left(D_{m}\right)}$ over $\mathscr{M}\left(D_{0}\right)$. 
Theorem 1. Let the notation and assumptions be as in Theorem $C$. Then we have

(1) $L_{p}(s ; \chi)\left(\chi \neq \chi^{0}\right)$ and $(s-1) L_{p}\left(s ; \chi^{0}\right)$ are elements of $\mathcal{O}\left(D_{0}\right)$.

(2) For each integer $m \geqq 0, L_{p}(s ; \chi)$ can be extended to an element of $\mathcal{O}\left(D_{m}\right)\left[T_{m}\right]$ if $\chi$ is not exceptional.

(3) Any $L_{p}(s ; \chi)$ with an exceptional character $\chi$ can be extended to an element of $(s-1)^{-1} \mathcal{O}\left(D_{m}\right)\left[T_{m}\right]$ for each integer $m \geqq 0$. Furthermore, if the order of $\chi\left(\gamma_{0}\right)$ is greater than $p^{m}$, than $L_{p}(s ; \chi)$ can be extended to an element of $\mathcal{O}\left(D_{m}\right)\left[T_{m}\right]$.

Proof. Let $G(T ; \chi)$ be as in Theorem $C$. If $\chi$ is not exceptional, then $G(T ; \chi)$ is an element of $\mathfrak{o}_{\chi}[[T]]$. Hence, by the corollary to Lemma 2, $L_{p}(s ; \chi)$ belongs to $\mathcal{O}\left(D_{0}\right)$ and it can be extended to an element of $\mathcal{O}\left(D_{m}\right)\left[T_{m}\right]$ for any integer $m \geqq 0$.

If $\chi$ is exceptional, then

$$
\left\{\chi^{-1}\left(\gamma_{0}\right) \kappa\left(\gamma_{0}\right)^{s}-\kappa\left(\gamma_{0}\right)\right\} L_{p}(s ; \chi)
$$

belongs to $\mathcal{O}\left(D_{0}\right)$ and it can be extended to an element of $\mathcal{O}\left(D_{m}\right)\left[T_{m}\right]$ for any integer $m \geqq 0$. If the order of $\chi\left(\gamma_{0}\right)$ is greater than $p^{m}$, then

$$
\begin{aligned}
\left|\chi\left(\gamma_{0}\right) \kappa\left(\gamma_{0}\right)-1\right| & =\left|\kappa\left(\gamma_{0}\right)\left(\chi\left(\gamma_{0}\right)-1\right)+\left(\kappa\left(\gamma_{0}\right)-1\right)\right| \\
& =\left|\chi\left(\gamma_{0}\right)-1\right| \geqq\left|p^{1 /(p-1) p^{m}}\right| .
\end{aligned}
$$

Hence, for any $T_{m}$ with $\left|T_{m}-1\right|<\left|p^{1 /(p-1) p^{m}}\right|$, we have

$$
\left\{T_{m}-\chi\left(\gamma_{0}\right) \kappa\left(\gamma_{0}\right)\right\}^{-1}=\sum_{n=0}^{\infty} \frac{\left(T_{m}-1\right)^{n}}{\left\{\chi\left(\gamma_{0}\right) \kappa\left(\gamma_{0}\right)-1\right\}^{n+1}} .
$$

Hence $\left\{\chi\left(\gamma_{0}\right)^{-1} T_{m}-\kappa\left(\gamma_{0}\right)\right\}^{-1}=\chi\left(\gamma_{0}\right)\left\{T_{m}-\chi\left(\gamma_{0}\right) \kappa\left(\gamma_{0}\right)\right\}^{-1}$ is an element of $\mathcal{O}\left(D_{m}\right)\left[T_{m}\right]$ (cf. Lemma 2). Hence $L_{p}(s ; \chi)$ can be extended to an element of $\mathcal{O}\left(D_{m}\right)\left[T_{m}\right]$ in this case. If the order of $\chi\left(\gamma_{0}\right)$ is not greater than $p^{m}$, then $\chi\left(\gamma_{0}\right)^{p^{m}}=1$. Since

$$
(s-1) \prod_{\zeta^{p^{m}=1}}\left\{\zeta T_{m}-\kappa\left(\gamma_{0}\right)\right\}^{-1}=\kappa\left(\gamma_{0}\right)^{-p^{m}}(s-1)\left\{\kappa\left(\gamma_{0}\right)^{p^{m(s-1)}}-1\right\}^{-1}
$$

is an element of $\mathcal{O}\left(D_{m}\right)\left[T_{m}\right],(s-1)\left\{\chi\left(\gamma_{0}\right)^{-1} \boldsymbol{T}_{m}-\kappa\left(\gamma^{0}\right)\right\}^{-1}$ is an element of $\mathcal{O}\left(D_{m}\right)\left[T_{m}\right]$ because $\zeta T_{m}-\kappa\left(\gamma_{0}\right) \in \mathcal{O}\left(D_{m}\right)\left[T_{m}\right]$ for any $\zeta$. Hence $L_{p}(s ; \chi)$ can be extended to an element of $(s-1)^{-1} \mathcal{O}\left(D_{m}\right)\left[T_{m}\right]$ in this case. Hence the theorem is proved.

Remark. J.-P. Serre found that $(s-1) L_{p}\left(s ; \chi^{0}\right)$ is an analytic function 
on $D_{0}$ by making use of the theory of $p$-adic modular forms (cf. Serre [17], p. 261). In this sense, the assertion (1) of Theorem 1 is essentially due to Serre.

Remark. If $F=Q$, then we can prove this theorem also by the method of Kubota-Leopoldt [11]. In fact, the author discovered the multivalued analytic continuation of the $p$-adic $L$-function in this way. With a slightly different method, $L$. Washington also discovered the multivalued analytic continuation of the $p$-adic $L$-function for the case $F=\boldsymbol{Q}$ (cf. Washington [18]).

\section{$\S 2$. The domain of analyticity of $L_{p}(s ; \chi)$}

Let $D$ be an open subset of $k$. We say that $D$ is quasi-connected iff, for any $x, y \in D$,

$$
\{|z-x||z \in k, z \notin D,| z-x|\leqq| x-y \mid\}
$$

is a finite set. M. Krasner constructed a theory of analytic functions on such a set (cf. Krasner [10], Robba [16], Morita [14]). Let $\mathcal{O}^{\prime}(D)$ be the set consisting of all Krasner analytic functions on $D$. Then $\mathcal{O}^{\prime}(D)$ is a $k$-algebra and an integral domain. Further $\mathcal{O}^{\prime}(D)$ is integrally closed in its quotient field $\mathscr{M}^{\prime}(D)$ (cf. Appendix).

Let $D$ be $\{z \in k|| z-a \mid<r\}(a \in k, 0<r)$ or $\left\{z \in k|r<| z-a \mid<r^{\prime}\right\}$ $\left(a \in k, 0 \leqq r \leqq r^{\prime}\right)$. Then $D$ is a quasi-connected set. It is known that the set $\mathcal{O}(D)$ consisting of all Taylor or Laurent series whose coefficients belong to $k$ and which converge on $D$ is an integral domain and integrally closed in its quotient field $\mathscr{M}(D)$. Further $\mathcal{O}(D)$ is contained in $\mathcal{O}^{\prime}(D)$, and, if $k$ is maximally complete, then $\mathcal{O}(D)=\mathcal{O}^{\prime}(D)$. (For the relation of Krasner analytic functions and rigid analytic spaces, see [14].)

Let $D$ be as above, and let $f$ be a function on $D$. Then we say that $f$ is an analytic function if $f$ belongs to $O(D)$. Further we say that $f$ has no analytic continuation outside $D$ if there exist no quasiconnected set $D^{\prime}$ and element $g$ of $\mathcal{O}^{\prime}\left(D^{\prime}\right)$ such that $D^{\prime} \supseteq D$ and $g(z)=f(z)$ holds for any point $z$ of $D$.

Let $\exp (z)$ be as in $\S 1$. Then, by making use of a $p$-adic analogue of the gap theorem, P. Robba proved that $\exp (z)$ has no analytic continuation outside the open disc $\left\{z \in k|| z|<| p^{1 /(p-1)} \mid\right\}$ (cf. Robba [16], Exemples 15.12). Let $\kappa\left(\gamma_{0}\right), D_{m}(m=0,1, \cdots)$ etc. be as in $\S 1$. Then, repeating the proof of Lemma 1, we obtain 
Lemma 3. (1) Lemma 1 holds even if we replace $D_{m}$ by $D_{m} \backslash\{1\}$.

(2) Let $D$ be a quasi-connected set satisfying $D \nsubseteq D_{m}$ and $D \cong D_{m+1}$. Then the equation

$$
T_{m+1}^{p^{m+1}}=\kappa\left(\gamma_{0}\right)^{p^{m+1}}
$$

of $T_{m+1}$ is irreducible over $\mathscr{M}^{\prime}(D)$. Hence any root $T_{m+1}$ of this equation generates over $\mathscr{M}^{\prime}(D)$ a cyclic extension of degree $p^{m+1}$.

Let $F$ be a totally real finite algebraic number field. Let $X=X_{F}$ be the set consisting of all characters of the Galois group of the maximal abelian extension $F_{a b}$ of $F$ over $F$ such that its kernel corresponds to a totally real finite algebraic number field. Then, by Theorem $\mathrm{C}$, we have the $p$-adic $L$-function $L_{p}(s ; \chi)$ for any $\chi \in X$. Let $\left\{L_{p}(s ; \chi)\right\}_{\chi \in X}$ be the vector with $L_{p}(s ; \chi)$ at the $\chi$-component. Let $f\left(\left\{L_{p}(s ; \chi)\right\}_{x \in X}\right)$ be a polynomial of the $L_{p}(s ; \chi)$. Let $\chi^{0}$ be the trivial character, and let $\pi$ be an exceptional character. Then $\chi \mapsto \pi \chi$ induces a permutation of $X$. Hence the polynomial $f\left(\left\{L_{p}(s ; \pi \chi)\right\}_{x \in X}\right)$ is well-defined.

Now we have the following theorem:

Theorem 2. Let $F, X, f\left(\left\{L_{p}(s ; \chi)\right\}_{x \in X}\right)$ be as above. Then this function of $s$ can be extended to an analytic function on $D_{m} \backslash\{1\}(m \geqq 0)$ iff

$$
f\left(\left\{L_{p}(s ; \pi \chi)\right\}_{x \in X}\right)=f\left(\left\{L_{p}(s ; \chi)\right\}_{x \in X}\right)
$$

holds for any exceptional character $\pi$ satisfying $\pi^{p^{m}}=\chi^{0}$. Furthermore, if $m$ is maximum with this property, then there exists no quasi-connected set $D$ properly containing $D_{m}$ such that $f\left(\left\{L_{p}(s ; \chi)\right\}_{x \in X}\right)$ can be extended to an element of $\mathcal{O}^{\prime}(D \backslash\{1\})$.

Proof. Since $1 /(s-1)$ belongs to $\mathcal{O}\left(D_{m} \backslash\{1\}\right)$, it follows from Theorem 1 that each $L_{p}(s ; \chi)$ can be extended to an element of $\mathcal{O}\left(D_{m} \mid\{1\}\right)\left[T_{m}\right]$. Hence $f\left(\left\{L_{p}(s ; \chi)_{x \in X}\right)\right.$ can be extended to an element of $\left.\mathcal{O}\left(D_{m}\right)\{1\}\right)\left[T_{m}\right]$.

Let $G(T ; \chi)$ be as in Theorem C. Then this element of $\mathcal{O}\left(D_{m} \mid\{1\}\right)\left[T_{m}\right]$ is given by $f\left(\left\{G\left(T_{m}-1 ; \chi\right)\right\}_{x \in X}\right)$. Hence, in view of the theorem of identity, the invariance in the theorem holds iff

$$
f\left(\left\{G\left(T_{m}-1 ; \pi \chi\right)\right\}_{x \in X}\right)=f\left(\left\{G\left(T_{m}-1 ; \chi\right)\right\}_{x \in X}\right)
$$

holds for any such characters $\pi$. Hence, by the corollary to Theorem C, this invariance holds iff $\left.f\left(G\left(T_{m}-1 ; \chi\right)\right\}_{x \in X}\right)$ is invariant under the conjugations $T_{m} \mapsto \pi\left(\gamma_{0}\right)^{-1} T_{m}$ of $\mathcal{O}\left(D_{m} \mid\{1\}\right)\left[T_{m}\right]$ over $\mathcal{O}\left(D_{m} \mid\{1\}\right)$. Hence the in- 
variance in the theorem is equivalent to the invariance under the automorphisms $T_{m} \mapsto \eta T_{m}\left(\eta^{p m}=1\right)$ of $\mathscr{M}\left(D_{m} \mid\{1\}\right)\left[T_{m}\right]$ over $\mathscr{M}\left(D_{m} \mid\{1\}\right)$. By Galois theory, this latter invariance holds iff $f\left(\left\{G\left(T_{m}-1 ; \chi\right)\right\}_{x \in X}\right)$ belongs to $\mathscr{M}\left(D_{m} \mid\{1\}\right)$ (cf. Lemma 3). Since $\mathcal{O}\left(D_{m} \mid\{1\}\right)$ is integrally closed in $\mathscr{M}\left(D_{m} \mid\{1\}\right)$ and since $f\left(\left\{G\left(T_{m}-1 ; \chi\right)\right\}_{x \in X}\right)$ is integral over $\mathcal{O}\left(D_{m} \backslash\{1\}\right)$, the invariance in the theorem holds iff $f\left(\left\{G\left(T_{m}-1 ; \chi\right)\right\}_{x \in X}\right)$ belongs to $\mathcal{O}\left(D_{m} \mid\{1\}\right)$. By the remark before Theorem 1, this proves the first assertion.

Now we assume that $m$ is maximum with this property. Let $D$ be a quasi-connected set properly containing $D_{m}$. Then $D \cap D_{m+1} \nsubseteq D_{m}$. Hence, by Lemma $3, T_{m+1}$ generates a cyclic extension of $\mathscr{M}^{\prime}\left(D \cap D_{m+1} \mid\{1\}\right)$ of degree $p^{m+1}$. Hence, repeating the above argument, we see that $f\left(\left\{G\left(T_{m+1}-1 ; \chi\right)\right\}_{x \in X}\right)$ does not belong to $\mathscr{M}^{\prime}\left(D \cap D_{m+1} \mid\{1\}\right)$. This shows that $f\left(\left\{L_{p}(s ; \chi)\right\}_{x \in X}\right)$ does not belong to $\mathcal{O}^{\prime}(D \backslash\{1\})$ because $\mathcal{O}^{\prime}(D \backslash\{1\}) \subseteq \mathscr{M}^{\prime}\left(D \cap D_{m+1} \mid\{1\}\right)$. Hence the theorem is proved.

Remark. Let $f\left(\left\{L_{p}(s ; \chi)\right\}_{x \in X}\right)$ and $m$ be as in Theorem 2. Then we have proved that $f\left(\left\{L_{p}(s ; \chi)\right\}_{x \in X}\right)$ has no meromorphic continuation outside $D_{m}$.

As for the $p$-adic $L$-function itself, we have

Theorem 3. Let $F$ and $X$ be as in Theorem 2. Let $\chi$ be an element of $X$. Then the p-adic L-function $L_{p}(s ; \chi)$ can be extended to an analytic function on $D_{m} \mid\{1\}(m \geqq 0)$ iff $L(s ; \pi \chi)=L(s ; \chi)$ holds for any exceptional character $\pi$ satisfying $\pi^{p^{m}}=\chi^{0}$. Furthermore, if $m$ is maximum with this property, then $L_{p}(s ; \chi)$ has no analytic continuation outside $D_{m}$ or $D_{m} \mid\{1\}$.

Proof. If $\chi$ is not $\chi^{0}$, then $L_{p}(s ; \chi)$ is an element of $\mathcal{O}\left(D_{0}\right) \subseteq \mathcal{O}^{\prime}\left(D_{0}\right)$. Let $D^{\prime}$ be a quasi-connected set containing 1 . Then $L_{p}(s ; \chi)$ is analytic on $D^{\prime}$ iff $L_{p}(s ; \chi)$ is analytic on $D^{\prime}\{\{1\}$ because it is analytic at 1 . Therefore, in view of Theorem 2 , this theorem follows from the following lemma.

Lemma 4. Let $F$ and $F^{\prime}$ be two real finite algebraic number fields. Let $\chi$ and $\chi^{\prime}$ be elements of $X_{F}$ and $X_{F^{\prime}}$. Then

$$
L_{p}(s ; \chi)=L_{p}\left(s ; \chi^{\prime}\right)
$$

holds iff

$$
L(s ; \chi)=L\left(s ; \chi^{\prime}\right) .
$$

Proof. If $L(s ; \chi)=L\left(s ; \chi^{\prime}\right)$, then, taking the $p$-parts of both series, we obtain 


$$
\prod_{p \mid p}\left(1-\chi(\mathfrak{p})(N \mathfrak{p})^{-s}\right)=\prod_{\mathfrak{p}^{\prime} \mid p}\left(1-\chi^{\prime}\left(\mathfrak{p}^{\prime}\right)\left(N \mathfrak{p}^{\prime}\right)^{-s}\right)
$$

where $\mathfrak{p}$ and $\mathfrak{p}^{\prime}$ are over prime ideals of $F$ and $F^{\prime}$ dividing $p$. Hence we have

$$
L_{S}(s ; \chi)=L_{S^{\prime}}\left(s ; \chi^{\prime}\right)
$$

where $S$ and $S^{\prime}$ are the sets consisting of such $\mathfrak{p}$ and $\mathfrak{p}^{\prime}$. By the definition of the $p$-adic $L$-functions, this implies that $L_{p}(s ; \chi)$ and $L_{p}\left(s ; \chi^{\prime}\right)$ are equal for any integer $s \leqq 0$ satisfying $s \equiv 1$ (modulo $(p-1)$ ) or (modulo 4 ) according as $p>2$ or $p=2$. Since the set of such integers has an accumlation point in $D_{0} \backslash\{1\}$, it follows from the theorem of identity (cf. Krasner [10]) that $L_{p}(s ; \chi)$ and $L_{p}\left(s ; \chi^{\prime}\right)$ are equal identically.

Now we assume that $L_{p}(s ; \chi)=L_{p}\left(s ; \chi^{\prime}\right)$. Then $L(s ; \chi)=L\left(s ; \chi^{\prime}\right)$ holds for any integers of the above form. By the functional equations, this implies

$$
\begin{aligned}
a_{\chi} b_{x}^{s}\{ & \left.\Gamma(1-s) \sin \left(\frac{\pi}{2} s\right)\right\}^{r} \prod_{\mathfrak{p} \in S}\left(1-\chi(\mathfrak{p})(N \mathfrak{p})^{-s}\right) \\
& \times \prod_{\mathfrak{r}^{\prime}}\left(1-\bar{\chi}(\mathfrak{l})(N \mathfrak{l})^{s-1}\right)^{-1} \\
= & a_{\chi^{\prime}} b_{\chi^{\prime}}^{s}\left\{\Gamma(1-s) \sin \left(\frac{\pi}{2} s\right)\right\}^{r^{\prime}} \prod_{\mathfrak{p}^{\prime} \in S^{\prime}}\left(1-\chi^{\prime}\left(\mathfrak{p}^{\prime}\right)\left(N \mathfrak{p}^{\prime}\right)^{-s}\right) \\
& \times \prod_{\mathfrak{l}^{\prime}}\left(1-\bar{\chi}^{\prime}\left(\mathfrak{l}^{\prime}\right)\left(N \mathfrak{l}^{\prime}\right)^{s-1}\right)^{-1},
\end{aligned}
$$

where $a_{x}, a_{x^{\prime}} \in C^{\times}, b_{x}, b_{x^{\prime}} \in \boldsymbol{R}^{\times}, \quad r=[F: Q], r^{\prime}=\left[F^{\prime}: Q\right]$, and $\mathfrak{l}$ and $\mathfrak{l}^{\prime}$ are over all prime ideals of $F$ and $F^{\prime}$. By the Stirling formula, we obtain $r=r^{\prime}$. Then we divide the both sides of (2.4) by $\{\Gamma(1-s) \sin ((\pi / 2) s)\}^{r}=$ $\{\Gamma(1-s) \sin ((\pi / 2) s)\}^{r^{\prime}} \neq 0$ and obtain

$$
\begin{aligned}
& a_{\chi} b_{\chi}^{s} \prod_{p \in S}\left(1-\chi(\mathfrak{p})(N \mathfrak{p})^{-s}\right) \prod_{\mathfrak{l}}\left(1-\bar{\chi}(\mathfrak{l})(N \mathfrak{l})^{s-1}\right)^{-1} \\
& \quad=a_{\chi^{\prime}} b_{\chi^{\prime}}^{s} \prod_{\mathfrak{p}^{\prime} \in S^{\prime}}\left(1-\chi^{\prime}\left(\mathfrak{p}^{\prime}\right)\left(N \mathfrak{p}^{\prime}\right)^{-s}\right) \prod_{\mathfrak{l}^{\prime}}\left(1-\bar{\chi}^{\prime}\left(\mathfrak{l}^{\prime}\right)\left(N \mathfrak{l}^{\prime}\right)^{s-1}\right)^{-1} .
\end{aligned}
$$

Here we expand the products in both sides in Dirichlet series and take $s$ sufficiently near $-\infty$. Then we see that (2.5) holds as an equality between functions of $s$. Hence we obtain

$$
a_{\chi} b_{x}^{s}=a_{x^{\prime}} b_{\chi^{\prime}}^{s}
$$

and 


$$
\begin{aligned}
\prod_{\mathfrak{p} \in S}\left(1-\chi(\mathfrak{p})(N \mathfrak{p})^{-s}\right) \prod_{\mathfrak{l}}\left(1-\bar{\chi}(\mathfrak{l})(N \mathfrak{l})^{s-1}\right)^{-1} \\
\quad=\prod_{\mathfrak{p}^{\prime} \in S^{\prime}}\left(1-\chi^{\prime}\left(\mathfrak{p}^{\prime}\right)\left(N \mathfrak{p}^{\prime}\right)^{-s}\right) \prod_{\mathfrak{l}}\left(1-\bar{\chi}^{\prime}\left(\mathfrak{l}^{\prime}\right)\left(N \mathfrak{I}^{\prime}\right)^{s-1}\right)^{-1},
\end{aligned}
$$

both as equalities between functions of $s$.

Let $\ell$ be any prime number different from $p$. Then, comparing the coefficients of $1 / \ell^{m s}(m=0,1, \cdots)$ of the both sides of (2.7), we obtain

$$
\prod_{1 \| \ell}\left(1-\bar{\chi}(I)(N I)^{s-1}\right)^{-1}=\prod_{l^{\prime} \mid \ell}\left(1-\bar{\chi}^{\prime}\left(I^{\prime}\right)\left(N I^{\prime}\right)^{s-1}\right)^{-1} \text {. }
$$

Hence $L_{S}(s ; \bar{\chi})$ and $L_{S^{\prime}}\left(s ; \bar{\chi}^{\prime}\right)$ are equal. Since $S$ and $S^{\prime}$ are finite sets, it is well-known that this implies $L(s ; \bar{\chi})=L\left(s ; \bar{\chi}^{\prime}\right)$ (for example, we can prove it by making use of the functional equations and repeating the above argument to the quotients of them). Restricting $s$ to $\boldsymbol{R}$ and taking the complex conjugation, we obtain $L(s ; \chi)=L\left(s ; \chi^{\prime}\right)$. Since $L(s ; \chi)$ and $L\left(s ; \chi^{\prime}\right)$ are analytic functions of $s$, this completes the proof of Lemma 4.

Remark. Let $L_{p}(s ; \chi)$ and $m$ be as in Theorem 3 . Let $n$ be any integer satisfying $n \geqq m$. Then, by Theorem $1, L_{p}(s ; \chi)$ can be extended to an element of $\mathcal{O}\left(D_{n} \mid\{1\}\right)\left[T_{n}\right]$. Hence there exists a polynomial $f(X)=$ $X^{k}+a_{1}(s) X^{k-1}+\cdots+a_{k}(s) \in \mathcal{O}\left(D_{n} \mid\{1\}\right)[X]$ such that $f(X)$ is irreducible over $\mathscr{M}^{\prime}\left(D_{n} \backslash\{1\}\right)$ and $f\left(L_{p}(s ; \chi)\right)=0$ for any $s \in D_{m}$. It is easy to see that $k=p^{n-m}$ and any root of $f(X)=0$ for $s \in D_{m}$ has the form $L_{p}(s ; \pi \chi)$ with a character $\pi$ of $\mathrm{Gal}\left(F_{n} / F_{m}\right)$. In this sense, the set $\left\{L_{p}(s ; \pi \chi)\right\}$ consisting of such functions gives a multivalued analytic function on $D_{n}$ in the sense of Krasner [10]. Furthermore, by Lemma 2, the parameter $T_{m}$ of Lemma 1 gives a unifomization of this multivalued function.

Hereafter we are going to study the condition in Theorem 3.

Let $F$ be a finite algebraic number field. Let $\chi$ and $\chi^{\prime}$ be characters of the Galois group of the maximal abelian extension $F_{a b}$ of $F$ over $F$ such that the kernels of them correspond to finite extensions of $F$. Let $M_{z}$ and $M_{x^{\prime}}$ be the corresponding fields. Hereafter in $\S 2$, for any finite algebraic number field $N$, we denote by $\tilde{N}$ the Galois closure of $N$ over $\boldsymbol{Q}$.

Now we assume

$$
L(s ; \chi)=L\left(s ; \chi^{\prime}\right) .
$$

Then, taking the $\ell$-part of the Dirichlet series expansions of them, we obtain

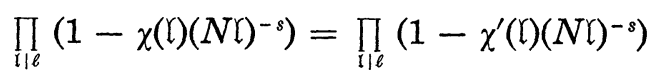


for any prime number $\ell$, where $\mathfrak{l}$ runs over all prime ideals of $F$ dividing $\ell$. Further (2.9) holds iff (2.10) holds for any prime number $\ell$ except a finite number of exceptions. We note that (2.10) is equivalent to

$$
\bigcup_{1 / \ell} \bigcup_{\zeta}\left\{\zeta \chi(l)^{1 / f(l)}\right\}=\bigcup_{1 l \ell} \bigcup_{\zeta}\left\{\zeta \chi^{\prime}(\mathfrak{l})^{1 / f(\mathfrak{l})}\right\},
$$

where the equality holds not only set theoretically but also with multiplicities, $f(\mathfrak{l})$ is the residue degree of $\mathfrak{l}$, and $\zeta$ runs over all $f(\mathfrak{l})$ th roots of unity. It follows from the density theorem that this equality implies $\tilde{M}_{\chi}=\tilde{M}_{\chi^{\prime}}$.

Since $M_{x}$ is a Galois extension of $F$, Gal $\left(M_{x} / F\right)$ is a homomorphic image of $\operatorname{Gal}\left(\tilde{M}_{\chi} / F\right)$. Hence $\chi$ induces a character of $\operatorname{Gal}\left(\tilde{M}_{\chi} / F\right)$. Since $\bigcup_{1 \mid \ell} \bigcup_{\zeta}\left\{\zeta \chi(l)^{1 / f(l)}\right\}$ coincides with the set of eigen values of the induced

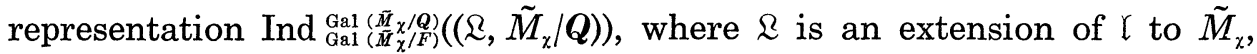
it follows from the density theorem that $L(s ; \chi)=L\left(s ; \chi^{\prime}\right)$ holds iff $\tilde{M}_{\chi}=$

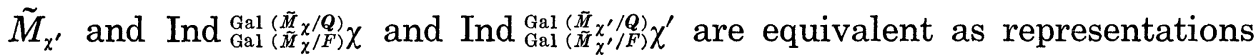
of $\operatorname{Gal}\left(\tilde{M}_{\chi} / \boldsymbol{Q}\right)=\operatorname{Gal}\left(\tilde{M}_{\chi^{\prime}} / \boldsymbol{Q}\right)$.

It is obvious that if $L(s ; \chi)=L\left(s ; \chi^{\prime}\right)$, then (1) $L\left(s ; \chi \chi^{\prime \prime}\right)=L\left(s ; \chi^{\prime} \chi^{\prime \prime}\right)$ for any character $\chi^{\prime \prime}$ of $\operatorname{Gal}\left(\boldsymbol{Q}_{a b} / \boldsymbol{Q}\right)$ and $(2) L\left(s ; \chi^{\alpha}\right)=L\left(s ; \chi^{\prime \alpha}\right)$ for any integer $\alpha$ prime to $[F: Q]$. On the other hand, let $\sigma$ be an automorphism of $M_{\chi}$ over $\boldsymbol{Q}$ satisfying $F^{\sigma}=F$. Let $\chi_{\sigma}(\tau)=\chi\left(\sigma^{-1} \tau \sigma\right)$ for any $\tau \in \operatorname{Gal}\left(M_{\chi} / F\right)$. Then $L(s ; \chi)=L\left(s ; \chi_{\sigma}\right)$ holds. Hence we can construct many examples of (2.9).

Of course, this problem is closely connected with the following wellknown problem: When do two Dedekind zeta functions coincide? For example, if $\left[M_{\chi}: F\right]=\left[M_{\chi^{\prime}}: F\right]=2$, then $L(s ; \chi)=L\left(s ; \chi^{\prime}\right)$ iff the zeta functions of $M_{\chi}$ and $M_{x^{\prime}}$ coincide. In particular, we can construct examples other that the above type (3) (cf. Perlis [15]).

Let $F, L(s ; \chi), \pi, \cdots$ be as in Theorem 3 . We assume that $m \geqq 1$ and

$$
L(s ; \chi)=L(s ; \pi \chi) \text {. }
$$

Let $M=M_{\chi}$ and $P=P_{\pi}$ be the fields corresponding to $\operatorname{ker}(\chi)$ and $\operatorname{ker}(\pi)$. Then it follows from the density theorem that the Galois closure $\tilde{M}$ of $M$ over $\boldsymbol{Q}$ contains $P$.

Let $\ell$ be a prime number such that $\ell$ remains prime in $P$. Then $\pi(\mathfrak{l})^{1 / f(\mathfrak{l})}=\pi(\ell)$ is a primitive $[P: Q]$ th root of unity and $\bigcup_{\mathbb{l} \mid \ell} \bigcup_{\zeta}\left\{\zeta \chi(\mathfrak{l})^{1 / f(\mathfrak{l})}\right\}=$ $\bigcup_{1 / \ell} \bigcup_{\zeta}\left\{\zeta \pi(\ell) \chi(l)^{1 / f(1)}\right\}$. Hence the group of $[P: Q]$ th roots of unity acts on the set $\left.\bigcup_{1} \bigcup_{\zeta}\{\zeta \chi(l))^{1 / f(1)}\right\}$ faithfully. Since this set consists of [F:Q] ele- 
ments, $\left[P_{\pi}: Q\right]$ divides $[F: Q]$.

Let $\ell$ be a prime number, and let $\alpha$ be the order of the group generated by the $\chi(l)$ for $\mathfrak{l} \mid \ell$. Then it follows that

$$
\bigcup_{i \mid \ell} \bigcup_{\zeta}\left\{\zeta^{\alpha}\right\}=\bigcup_{\| l \ell} \bigcup_{\zeta}\left\{\pi(\ell)^{\alpha} \zeta^{\alpha}\right\}
$$

If $\pi(\ell)^{\alpha}$ is not 1 , let $p^{\beta}$ be the order of $\pi(\ell)$. If $p^{\beta}$ divides $f(l)$, then the group of all $f(l)$ th roots of unity contains $\pi(\ell)$. Hence $\bigcup_{\zeta}\left\{\zeta^{\alpha}\right\}=\bigcup_{\zeta}\left\{\pi(\ell)^{\alpha} \zeta^{\alpha}\right\}$. If $p^{\beta}$ does not divide $f(\mathfrak{l})$, then the $p$-part of the order of $\pi(\ell)^{\alpha}$ is greater than the $p$-part of the order of any $\zeta^{\alpha \alpha}\left(\zeta^{\prime f(l)}=1\right)$. Since this contradicts (2.13), it follows that $\pi(\ell)^{\alpha}=1$ or the order of $\pi(\ell)$ divides $f(l)$ for any prime ideal $\mathfrak{l}$ dividing $\ell$. In particular, if $\mathfrak{l}$ is a prime ideal of $F$ such that $\mathfrak{l} \ell$ and $f(\mathfrak{l})=1$, then $\pi(\mathfrak{l})^{\alpha}=\pi(\ell)^{\alpha}=1$. Since $\alpha$ divides $[M: F], \pi(\mathfrak{l})^{[M: F]}=$ 1. By the density theorem, there exists a prime ideal $\mathfrak{l}$ such that $f(\mathfrak{l})=1$ and $\pi(\mathfrak{l})$ is a primitive $[F \cdot P: F]$ th root of unity. Since $[F \cdot P: F]=\left[P: P_{e}\right]$, this shows that $\left[P_{x}: P_{e}\right]$ divides $\left[M_{x}: F\right]$.

Since there exists $\pi$ such that $P_{\pi}=P_{m+e}$, we have proved

Theorem 4. Let $F$ and $X$ be as in Theorem 3. If the p-adic L-function $L_{p}(s ; \chi)(\chi \in X)$ can be extended to an analytic function on $D_{m} \mid\{1\}(m \geqq 0)$, then (1) the Galois closure $\tilde{M}_{\chi}$ of the field $M_{\chi}$ corresponding to $\operatorname{ker}(\chi)$ contains $P_{m+e}$, (2) $p^{m}$ divides $\left[M_{\chi}: F\right]$ and (3) $p^{m+e}$ divides $[F: Q]$. Further, if $L_{p}(s ; \chi)$ is analytic on $D_{m} \backslash\{1\}$, and if (4) $\tilde{M}_{\chi}$ does not contain $P_{m+e}$ or (5) $p^{m+1}$ does not divide $\left[M_{\chi}: F\right]$ or $(6) p^{m+e+1}$ does not divide $[F: Q]$, then $L_{p}(s ; \chi)$ has no analytic continuation outside $D_{m}$ or $D_{m} \backslash\{1\}$.

If $F=\boldsymbol{Q}$, then $p$ does not divide $[F: Q]$. Hence

Corollary 1. The p-adic L-function $L_{p}(s ; \chi)$ for a primitive Dirichlet character $\chi$ satisfying $\chi \neq \chi^{0}$ and $\chi(-1)=1$ has no analytic continuation outside $D_{0}=\left\{s \in k|| s|<| q^{-1} p^{1 /(p-1)} \mid\right\}$.

Remark. J. Fresnel proved that the Taylor expansion of $L_{p}(s ; \chi)$ converges exactly on $D_{0}$ by studying the Bernoulli numbers (cf. Fresnel [8]).

If $\chi=\chi^{0}$, then $p$ does not divide $\left[M_{\chi}: F\right]$. Hence

Corollary 2. Let $F$ be a totally real finite algebraic number field. Then the-padic zeta function $L_{p}\left(s ; \chi^{0}\right)$ of $F$ has no analytic continuation outside $D_{0}$ or $D_{0} \backslash\{1\}$. 
ExAmple. Let $p=2$ and $P=Q(\sqrt{2})$. Let $F$ be a real quadratic number field, and let $M$ be a cyclic extension of $F$ of order 4 . We assume that $F \neq P, M \supseteq P, M$ is totally real and $M$ is a non-abelian Galois extension

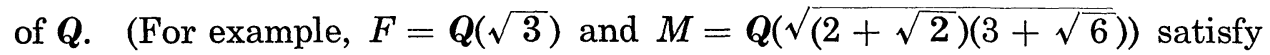
such conditions (cf. Dedekind [6].)) Let $\chi$ be a faithful character of $\operatorname{Gal}(M / F)$, and let $\pi$ be the Legendre character $\pi()=(\mid Q(\sqrt{2}))$.

Let $\ell$ be a prime number which is unramified in $M$.

If $\ell$ remains prime in $F$, let $\ell=\mathfrak{l}$ in $F$. Then $\pi(\mathfrak{l})=\pi((\mathfrak{l}, M / F))=$ $\pi((\ell, M / Q))^{2}=\pi((\ell, P / Q))^{2}=1$. Hence $\chi(\mathfrak{l})=\pi(\mathfrak{l}) \chi(\mathfrak{l})$. Therefore $\{ \pm \chi(\mathfrak{l})\}=$ $\{ \pm \pi(\mathfrak{l}) \chi(\mathfrak{l})\}$.

If $l$ is decomposed in $F$, let $\ell=\mathfrak{l}_{1} \mathfrak{l}_{2}$ in $F$. Let $i=1$ or 2 . If $\pi\left(\mathfrak{l}_{i}\right)=$ $\pi(l)=1$, then $\left\{\chi\left(\mathfrak{l}_{1}\right), \chi\left(\mathfrak{l}_{2}\right)\right\}=\left\{\pi\left(\mathfrak{l}_{1}\right) \chi\left(\mathfrak{l}_{1}\right), \pi\left(\mathfrak{l}_{2}\right) \chi\left(\mathfrak{l}_{2}\right)\right\}$. If $\pi\left(\mathfrak{l}_{i}\right)=\pi(l)=-1$, then $l_{i}$ remains prime in $F(\sqrt{2}) / F$. Since $\operatorname{Gal}(M / F)$ is a cyclic group, $\mathfrak{l}_{i}$ remains prime in $M / F$. Hence $\chi\left(\bigvee_{i}\right)=\chi\left(\left(\Upsilon_{i}, M / F\right)\right)$ is a primitive fourth root of unity. Since $M$ is not an abelian extension of $\boldsymbol{Q}$, the non-trivial automorphism of $F$ over $\boldsymbol{Q}$ acts non-trivially on $\operatorname{Gal}(M / F)$. Hence $\left(\mathfrak{l}_{1}, M / F\right) \neq$ $\left(\mathfrak{l}_{2}, M / F\right)$. Therefore $\chi\left(\left(\mathfrak{l}_{1}, M / F\right)\right) \neq \chi\left(\left(\mathfrak{l}_{2}, M / F\right)\right)$. Therefore $\chi\left(\mathfrak{l}_{2}\right)=\pi\left(\mathfrak{l}_{1}\right) \chi\left(\mathfrak{l}_{1}\right)$ and $\chi\left(\mathfrak{I}_{1}\right)=\pi\left(\mathfrak{I}_{2}\right) \chi\left(\mathfrak{I}_{2}\right)$. Hence $\left\{\chi\left(\mathfrak{I}_{1}\right), \chi\left(\mathfrak{l}_{2}\right)\right\}=\left\{\pi\left(\mathfrak{I}_{1}\right) \chi\left(\mathfrak{Y}_{1}\right), \pi\left(\mathfrak{I}_{2}\right) \chi\left(\mathfrak{I}_{2}\right)\right\}$.

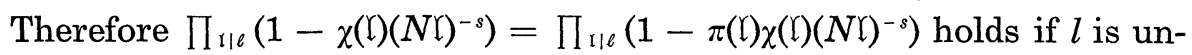
ramified in $M$. Therefore $L(s ; \chi)=L(s ; \pi \chi)$. By Theorem $3, L_{p}(s ; \chi)$ is an analytic function on $D_{1}$. Furthermore, since $[F: Q]=2, L_{p}(s ; \chi)$ has no analytic continuation outside $D_{1}$. We note that $D_{1}=\left\{s \in k|| s|<| p^{-1} q^{-1} p^{1 /(p-1)} \mid\right\}$ and $F \cap P=Q$ in this case.

\section{Appendix}

Let $k$ be an algebraically closed field with a non-trivial non-archimedean valuation $\mid 1$. We assume that $k$ is complete with respect to this valuation. Let $D$ be a quasi-connected set in $k$ or $\boldsymbol{P}^{1}(k)$, and let $\mathcal{O}^{\prime}(D)$ be the $k$-algebra of Krasner analytic functions on $D$. Since $\mathcal{O}^{\prime}(D)$ is a domain of integrity, we denote by $\mathscr{M}^{\prime}(D)$ the quotient field of $\mathcal{O}^{\prime}(D)$. Then

TheOREM. $\mathcal{O}^{\prime}(D)$ is integrally closed in $\mathscr{M}^{\prime}(D)$.

Proof. Let $f$ be an element of $\mathscr{M}^{\prime}(D)$ which is integral over $\mathcal{O}^{\prime}(D)$. Then there are elements $g, h, a_{1}, \cdots, a_{n}$ of $\mathcal{O}^{\prime}(D)$ such that $h \neq 0, f=g / h$, and

$$
f^{n}+a_{1} f^{n-1}+\cdots+a_{n}=0
$$


It is known that $D^{\times}=\{z \in D \mid h(z) \neq 0\}$ is quasi-connected and $1 / h$ is a Krasner analytic function on $D^{\times}$(cf. Krasner [10], p. 156).

Let $x$ be a point of $D$ such that $h(x)=0$. Since $h \neq 0$, it follows from the theorem of identity that there exists a ball $B$ such that $x \in B$, $B \subseteq D$ and $\{z \in B \mid h(z)=0\}=\{x\}$. By the definition of Krasner analytic functions, there exists a ball $B^{\prime}$ such that $x \in B^{\prime}, B^{\prime} \subseteq B$, and $g, h, a_{1}, \cdots, a_{n}$ are analytic elements on $B^{\prime}$. Then the restrictions of $g, h, a_{1}, \cdots, a_{n}$ are given by convergent power series. Since

$$
g^{n}=-h\left(a_{1} g^{n-1}+a_{2} g^{n-2} h+\cdots+a_{n} h^{n-1}\right),
$$

we see that $g$ has a zero at $z=x$ and the order of zero of $g$ at $z=x$ is not smaller than the order of zero of $h$ át $z=x$. Hence there exists a ball $B^{\prime \prime}=B^{\prime \prime}(x)$ such that $x \in B^{\prime \prime}$, the diameter of $B^{\prime \prime}$ belongs to $\left|k^{\times}\right|$, and $f=g / h$ is given by a convergent power series on $B^{\prime \prime}$. Then the restriction of $f$ to $B^{\prime \prime}$ is an analytic element. By the definition of Krasner analytic functions, $f$ is a Krasner analytic function on $D^{\times} \cup \cup_{x} B^{\prime \prime}(x)=D$. Hence the theorem is proved.

\section{REFERENCES}

[1] Amice, Y. et Fresnel, J., Fonctions zêta $p$-adiques des corps de nombres abéliens réels, Acta Arith., 20 (1972), 353-384.

[2] Cassou-Noguès, P., Valeurs aux entiers négatifs des fonctions zêta et fonctions zêta $p$-adiques, to appear.

[ 3 ] Coates, J., $P$-adic $L$ functions and Iwasawa's theory, Proceedings of the 1975 Durham Symposium, pp. 269-353. Academic Press, London-New York, 1977.

[ 4 ] Coates, J. and Sinnot, W., On $p$-adic $L$-functions over real quadratic fields, Inventiones math., 25 (1974), 253-279.

[5] - Integrality properties of the values of partial zeta functions, Proc. London Math. Soc., 34 (1977), 365-384.

[6] Dedekind, R., Gesammelte mathematische Werke I-III, Braunschweig, 1930-32.

[ 7 ] Deligne, P. and Ribet, K., Values of abelian $L$-functions at negative integers, to appear.

[8] Fresnel, J., Nombres Bernoulli et fonctions $L$ p-adiques, Ann. Inst. Fourier, 97 (1967), 281-333.

[ 9 ] Iwasawa, K., Lecture on $p$-adic $L$-functions, Ann. of Math. Studies, 74, Princeton U.P., Princeton, 1972.

[10] Krasner, M., Rapport sur le prolongement analytiques dans les corps valués complets par la methode des éléments analytiques quasi-connexes, Bull. Soc. Math. France, Mémoire, 39-40 (1974), 131-254.

[11] Kubota, T. and Leopoldt, H. W., Eine $p$-adiche Theorie der Zetawerte, I, J. reine u. angew. Math., 214-215 (1964), 328-339.

[12] Leopoldt, H. W., Eine $p$-adische Theorie der Zetawerte, II, J. reine u. angew. Math., 274/275 (1975), 224-239.

[13] Morita, Y., Analytic functions on an open subsets of $\boldsymbol{P}^{1}(k)$, to appear. 
[14] —- Krasner's analytic functions and rigid analytic spaces, to appear.

[15] Perlis, R., On the class numbers of arithmetically equivalent fields, to appear.

[16] Robba, P., Fonctions analytiques sur les corps valués ultramétriques complets, I, Astérisque, 10 (1973), 109-218.

[17] Serre, J.-P., Formes modulaires et fonctions zêta $p$-adiques, Lecture Notes in Math., 350 (1973), 191-268.

[18] Washington, L., Euler factors for $p$-adic $L$-functions, to appear.

Department of Mathematics

Hokkaido University 